\title{
Infections Musculosquelettiques De L'adulte AU Centre Hospitalier Universitaire De Brazzaville: Difficultés Diagnostiques Et De Prise En Charge
}

\author{
Angalla Affleck Romaric Ledier
}

Lamini N'Soundaht Norbert Edgard

Richard Bileckot

Ntsiba Honoré

Service de Rhumatologie, Centre Hospitalier Universitaire de Brazzaville, Congo

Faculté des Sciences de la Santé, Université Marien Ngouabi,

Brazzaville, Congo

Moyikoua Régis Franck

Service d'imagerie Médicale, Centre Hospitalier Universitaire de

Brazzaville, Congo

Faculté des Sciences de la Santé, Université Marien NgouabI, Brazzaville, Congo

\section{Bouhelo Pam Kevin Parfait Bienvenu}

Service de Traumatologie Orthopédie, Centre Hospitalier Universitaire de Brazzaville, Congo

Faculté des Sciences de la Santé, Université Marien Ngouabi, Brazzaville, Congo

\section{Akoli Ekoya Odzala \\ Nkouala Kidede Chabel \\ Omboumahou Bakale Francina \\ Salemo Anna \\ Kaloulou Armande}

Service de Rhumatologie, Centre Hospitalier Universitaire de Brazzaville, Brazzaville, Congo

\section{Doi:10.19044/esj.2021.v17n25p325}

Submitted: 31 May 2021

Accepted: 01 July 2021

Published: 31 July 2021
Copyright 2021 Author(s)

Under Creative Commons BY-NC-ND 4.0 OPEN ACCESS

Cite As:

Ledier A.A.R., Norbert Edgard L.N., Bileckot R., Honoré N., Régis Franck M., Bienvenu B.P.K.P., Odzala A.E., Chabel N.K., Francina O.B., Anna S. \& Armande K. (2021). Infections Musculosquelettiques De L'adulte AU Centre Hospitalier Universitaire De Brazzaville: 
Difficultés Diagnostiques Et De Prise En Charge. European Scientific Journal, ESJ, 17(25), 325.

https://doi.org/10.19044/esj.2021.v17n25p325

\section{Resumé}

Objectif: Rapporter les difficultés diagnostiques et de prise en charge des infections musculosquelettiques.

Patients et méthodes: Etude rétrospective menée dans les services de Rhumatologie et de Traumatologie/Orthopédie du CHU de Brazzaville du 1er Janvier 2017 au 30 Septembre 2020. Nous avons colligé et analysé les dossiers des patients hospitalisés pour infections musculosquelettiques. Le diagnostic d'infection musculosquelettique était retenu sur critères de Wald Vogel et al.

Résultats: La fréquence hospitalière était de 1.9\%. Les hommes prédominaient (54.8\%) avec un sex-ratio de 1.2. L'âge moyen était de 41.7ans (extrêmes 17 et 77 ans). Treize de nos patients étaient les travailleurs indépendants , 12 sans-emploi, les salariés et les étudiants respectivement dans 9 et 8 cas. La majorité de nos patients provenait du milieu rural dans 29 cas (69.1\%). Il s’agissait de 13 cas (30.9\%) d’ostéomyélites/Ostéites, 12 cas (28.5\%) d'arthrites infectieuses, 9 cas (21.4\%) de spondylodiscites tuberculoses, 4 cas (9.6\%) d'infections sur matériel d'ostéosynthèse et les myosites suppurées. Seuls 6 patients (14\%) avaient réalisé une imagerie en coupes (l'IRM dans 4 cas ,la TDM dans 2 cas), avec un délai moyen de réalisation de 6,1 jours (extrêmes 4 et 10 jours) et l'échographie ostéoarticulaire dans 6 cas (14\%). L'analyse bactériologique était effectuée dans 25 cas (59.5\%), parmi eux, une Pyo culture dans 11 cas (44\%), l'analyse du liquide articulaire dans 5 cas (20\%), les hémocultures 2 cas et la biopsie osseuse dans 1 cas(4\%), avec un délai moyen de réalisation de 5,8 jours (extrêmes 2 et 11 jours). La culture était positive dans 13 cas (52\%). Les principaux germes isolés étaient : le streptocoque dans 5 cas (38.4\%), le staphylocoque 3 cas (23.1\%), l'entérobactérie et le Pseudomonas dans 2cas (15.4\%), le bacteroides dans 1 cas $(7.7 \%)$.

Conclusion: Les infections musculosquelettiques sont une urgence diagnostique et thérapeutique et de diagnostic souvent tardif. Les principales difficultés sont le bas niveau socioéconomique et la faible accessibilité du plateau technique.

Mots-clés: Infection, Arthrite, Ostéite, Spondylodiscite, Myosite, Congo Brazzaville 


\title{
Musculoskeletal Infections In Adults At The Brazzaville University Hospital: Diagnostic And Management Difficulties
}

\section{Angalla Affleck Romaric Ledier}

Lamini N'Soundaht Norbert Edgard

Richard Bileckot

Ntsiba Honoré

Service de Rhumatologie, Centre Hospitalier Universitaire de Brazzaville, Congo

Faculté des Sciences de la Santé, Université Marien Ngouabi,

Brazzaville, Congo

Moyikoua Régis Franck

Service d'imagerie Médicale, Centre Hospitalier Universitaire de

Brazzaville, Congo

Faculté des Sciences de la Santé, Université Marien NgouabI, Brazzaville, Congo

\section{Bouhelo Pam Kevin Parfait Bienvenu}

Service de Traumatologie Orthopédie, Centre Hospitalier Universitaire de Brazzaville, Congo

Faculté des Sciences de la Santé, Université Marien Ngouabi, Brazzaville, Congo

\author{
Akoli Ekoya Odzala \\ Nkouala Kidede Chabel \\ Omboumahou Bakale Francina \\ Salemo Anna \\ Kaloulou Armande
}

Service de Rhumatologie, Centre Hospitalier Universitaire de Brazzaville, Brazzaville, Congo

Abstract

Objective: This paper focuses on reporting the difficulties in diagnosing and managing musculoskeletal infections.

Patients and Methods: Retrospective study was conducted in the Rheumatology and Traumatology/Orthopedics departments of the Brazzaville University Hospital from January 1st, 2017 to September 30th, 2020. The files of hospitalized patients were collected and analyzed for musculoskeletal infections. The diagnosis of musculoskeletal infection was made according to the criteria of Wald Vogel et al.

Results: The hospital frequency was $1.9 \%$. Men predominated (54.8\%) with a sex ratio of 1.2. The average age was 41.7 years (range 17 and 77). Thirteen of the patients were self-employed, while 12 were unemployed. There were also 9 employees and 8 students. The majority of patients came 
from rural areas in 29 cases (69.1\%). These were 13 cases (30.9\%) of osteomyelitis/Osteitis, 12 cases (28.5\%) of infectious arthritis, 9 cases (21.4\%) of tuberculosis spondylodiscitis, 4 cases $(9.6 \%)$ of infections on osteosynthesis material and suppurative myositis. Only 6 patients (14\%) had performed sectional imaging (MRI in 4 cases, CT in 2 cases), with an average completion time of 6.1 days (range 4 and 10 days). There were also 6 cases of ultrasound osteoarticular (14\%). Bacteriological analysis was performed in 25 cases (59.5\%): Pyo culture in 11 cases (44\%), joint fluid analysis in 5 cases (20\%), blood cultures in 2 cases and bone biopsy in 1 case (4\%), with an average completion time of 5.8 days (range 2 and 11 days). Culture was positive in 13 cases (52\%). The main germs isolated were streptococcus in 5 cases (38.4\%), staphylococcus in 3 cases (23.1\%), enterobacteria and Pseudomonas in 2 cases (15.4\%), and bacteroides in 1 case ( $7.7 \%)$.

Conclusion: Musculoskeletal infections are a diagnostic and therapeutic emergency which is often late in diagnosis. The main difficulties are the low socioeconomic level and the poor accessibility of the technical platform.

Keywords: Infection, Arthritis, Osteitis, Spondylodiscitis, Myositis, Congo Brazzaville

\section{Introduction}

Les infections musculosquelettiques regroupent un nombre important de situations cliniques : arthrites septiques ; spondylodiscites infectieuses ; ostéomyélites ; infections sur prothèse articulaire ; infections du pied diabétique ; ostéites post-opératoires avec ou sans matériel d'ostéosynthèse et les pyomyosites (Habett El khalil, 2018). Ces infections constituent une urgence diagnostique et thérapeutique, non seulement du fait des risques septiques, mais également des risques fonctionnels encourus notamment par la destruction des structures articulaires. Le diagnostic repose sur un trépied : clinique, biologique et imagerie, qui doit être précoce afin d'adapter le traitement (Weston et al., 1999). Les germes en cause sont très variés, les plus retrouvés sont : le staphylocoque aureus, le streptocoque et les bacilles à gram négatif, mais aussi le bacille de Koch (Asseray et al., 2012; Malghem et al., 1997). L’imagerie avec l'apport des différentes techniques telle que l'imagerie par résonance magnétique (IRM), la tomodensitométrie et la polymerase chain reaction (PCR) ont un rôle primordial dans le diagnostic précoce et dans la surveillance sous traitement. Elles intéressent toutes les tranches d'âge et leurs manifestations cliniques sont fonction du terrain, de la localisation, du germe responsable (Asseray et al., 2012). L'enquête bactériologique est cruciale avant toute antibiothérapie pour le succès thérapeutique (Dubost et al., 2000; Gupta et al., 2001; Ledantec et al., 1996; Studahl et al., 1994). La chirurgie, comprenant un lavage, un débridement une synovectomie, une 
séquestrectomie couplée à une antibiothérapie, paraît avantageuse par rapport au traitement médical seul (Bussiere et al., 1999; Faraj et al., 2002; Jerosh et al., 1995; Jerosh et al., 1998; Visposeara et al., 2002). En Afrique subsaharienne, les infections musculosquelettiques sont très fréquentes (Houzou et al., 2017; Moyikoua et al., 1993). Elles constituent une cause fréquente de morbidité à l'origine de handicap parfois sévère mais aussi de mortalité (Ntsiba et al., 2003). Au Congo Brazzaville, les infections musculosquelettiques constituent un motif fréquent d'hospitalisation dans le service de Rhumatologie au CHU-B avec une fréquence hospitalière de 33\% (Ntsiba et al., 2003). Plusieurs études ont été faites sur les infections musculosquelettiques parmi lesquelles 100 cas d'arthrites septiques du genou en zone tropicale (Ntsiba et al., 2003), les aspects morphologiques du Mal de POTT chez l'adulte congolais à Brazzaville (Lamini et al., 2019), les ostéomyélites chroniques de l'adulte à Brazzaville (Monka et al., 2018), aspects bactériologiques et évolutifs des arthrites septiques à Brazzaville (Biléckot et al., 2006). Dans le souci de compléter les travaux sur les infections musculosquelettiques en Afrique subsaharienne, particulièrement au Congo, nous avons initié cette étude dont l'objectif était de rapporter les difficultés diagnostiques et de prise en charge des infections musculosquelettiques au CHU de Brazzaville.

\section{Patients et Méthodes}

\section{Cadre et periode d'étude}

Il s'agissait d'une étude rétrospective, transversale, menée dans les services de Rhumatologie et deTraumatologie/Orthopédie du Centre Hospitalier Universitaire de Brazzaville (CHU-B), du 1er Janvier 2017 au 30 Septembre 2020, soit une période de 3 ans et 8 mois.

\section{Collecte et analyse des données}

Dans un premier temps, la collecte des données s'est faite sur les dossiers colligés des patients suivis pour infections musculosquelettiques. Ceux dont le diagnostic d'infections musculosquelettiques répondait aux critères de critères de Wald Vogel et al ont été retenus. A partir des données recueillies dans les dossiers médicaux, les patients ont été contactés individuellement par téléphone ou joints à leur domicile. Après consentement éclairé, les patients ont été vus en consultation dans le service de Rhumatologie du CHU, afin de recueillir les caractéristiques sociodémographiques (âge, sexe, profession, provenance), de rapporter les aspects cliniques et les difficultés diagnostiques et de prise en charge . 


\section{Criteres diagnostiques}

Parmi les patients hospitalisés, nous avons recherché, sur la base des données anamnestiques, cliniques, biologiques, radiologiques et histologiques, les signes d'infections musculosquelettiques :

\section{-données cliniques}

Le diagnostic d'infections musculosquelettiques était évoqué chez les patients présentant une fièvre, une porte d'entrée, une altération de l'état général, un terrain prédisposé, une fistule, une rachialgie inflammatoire, une tuméfaction des parties molles, des signes inflammatoires locaux avec une douleur, une tuméfaction, une augmentation de la chaleur locale, une raideur ou une gibosité,une monoarthrite, une oligoarthrite ou une polyarthrite fébrile;

\section{-données paracliniques}

-L'isolement d'un agent microbien par ponction articulaire, d'un abcès para vertébral, ponction de myosite, au niveau de la porte d'entrée, par les hémocultures, la pyoculture, l'uroculture ou coproculture et histologiquement par la mise envidence du germe à la biopsie synoviale , discovertébrale , osseuse et de la collection des parties molles ;

- Présence d'un syndrome inflammatoire biologique avec l'accélération de la vitesse de sédimentation (normale inférieure à $20 \mathrm{~mm}$ à la première heure), l'augmentation de la C-réactive protéine (normale inférieure à $6 \mathrm{mg} / \mathrm{l}$ ), l'hyperleucocytose à polynucléaires neutrophiles (normale comprise entre 4000 et 10000 globules blancs par millimètre de sang et 1800-7000 polynucléaires neutrophiles par millimètre cube) et une thrombocytose (normale 150.000 et 400.000 cellules par millimètre cube);

- sur le plan radiologique, l'existence de signes d'arthrite avec une déminéralisation epiphysaire, un pincement globale de l'interligne articulaire, des érosions ou géodes en miroir et des signes de spondylodiscite avec un pincement discal global, une déminéralisation des plateaux vertébraux adjacents, des érosions ou des géodes en miroir , l'apposition périostée, le séquestre, l'épaississement des parties molles ;

- A L'IRM par la presence d'une hyposignal en T1, une hypersignal en séquence STIR ou T1 après injection du gadolinium, l'hypersignal en T2 du disque et des plateaux adjacents avec abcès des parties molles paravertébrales et dans l'espace péridural, l'absence de rehaussement du signal du disque après injection de gadolinium en cas de tuberculose dans la spondylodiscite. Dans l'ostéite et l'ostéomyélite, un signal inflammatoire de la médullaire osseuse, bien avant que n'apparaissent les images radiographiques ou scannographiques et la mise en évidence d'un abcès des parties molles. Dans les arthrites, elle explore les cartilages articulaires, la synoviale, la trame ostéomédullaire et les structures musculotendinoligamentaires. 
- Tomodensitométrie (TDM) : l'érosion d'un angle vertébral, l'abcès péri vertébral ou épidural dans la spondylodiscite. Dans l'ostéite et l'ostéomyélite, le scanner a permis une étude fine de la corticale osseuse et la visualisation de la diffusion dans les parties molles et par la Présence d'un épanchement à l'échographie.

\section{Données thérapeutiques et évolutives}

- sur le plan évolutif et thérapeutique, guérison sous antibiothérapie.

\section{Criteres d'incluiosn}

Les patients agés d'au moins 17ans, hospitalisés pour infections musculosqueltttiques

\section{Criteres de non inclusion}

Ont été insclus ,les patients dont les dossiers médicaux ne comportaient aucun bilan minimum biologique ou radiologique, les patients hospitalisés pour autres affections, les infections chez l'enfant et les infections du pied diabétique .

Le logiciel SPSS 22 a permis l'analyse des données cliniques.

\section{Résultats}

\section{Données sociodémographiqueses}

Du 1er Janvier 2017 au 30 Septembre 2020, 2203 patients ont été admis dans les services de Rhumatologie et Traumatologie-Orthopédie du Centre Hospitalier Universitaire de Brazzaville, parmi lesquels 42 ont été hospitalisés pour infections musculosquelettiques soit une fréquence hospitalière de $1.9 \%$.). Notre échantillon était composé de 23 patients du sexe masculin (54.8\%) et 19 de sexe féminin (45.2\%). Le sex-ratio était de 1.2. La moyenne d'âge était de 41.6 ans avec des extrêmes de 17 et 77 ans. La tranche d'âge de 51 et 60 ans était la plus représentée (Figure 1).

La majorité de nos patients étaient les travailleurs indépendants dans 13 cas $(30.1 \%)$ suivi des sans-emplois 12 cas (28.4\%), les salariés et les étudiants respectivement dans 9 cas (21.4\%) et 8 cas (19.1\%). 29 patients (69.1\%) provenaient du milieu urbain et 13 (30.9\%) du milieu rural.

\section{Données cliniques}

\section{Types d'infections musculosquelettiques}

Les principales infections musculosquelettiques (Tableau I) retrouvées étaient : l'ostéomyélite/ostéite dans 13 cas (30.9\%), l'arthrite septique dans 12 cas (28.5\%) dont 10 à germes banals et 2 cas à germe specfique ( presumées tuberculeuses), la spondylodiscite infectieuse 
presumées tuberculeuses dans 9 cas (21.4\%), la myosite et les infections sur matériel dans 4 cas $(9.6 \%)$ respectivement.

\section{Delai de diagnostic}

Le delai moyen de diagnostic était de 27 jours avec des extremes de 5223 jours, avec $63,4 \%$ des cas au stade de chronicité.

\section{Motif de consulataion}

La douleur était le motif de consultation le plus retrouvé. Elle était présente chez tous les patients (100\%), suivie de l'impotence fonctionnelle dans 38 cas (90.5\%) et la fièvre chez 19 patients (45.2\%).

\section{Mode de debut}

Le debut des symptomes était progressif chez 27 patients (64.3\%) et brutal chez 15 patients (35.7\%). L'horaire des douleurs était inflammatoire chez 31 patients (73.8\%), mixte chez six (14.3\%) et mécanique chez cinq sujets $(11.9 \%)$.

\section{Examen clinique}

\section{Signes généraux}

L’état général était conservé chez 37 patients (88.1\%) et altéré chez 5 patients (11.9\%).

\section{Signes physiques}

Au plan locomoteur, les signes locaux retrouvées sont illustrés dans le tableau II. Au plan radiculaire, un patient avait une radiculalgie L5 droite. Une complication neurologique a été notée chez quatre patients (9.5\%) à type de compression médullaire respectivement au niveau C7-T2, T4-T5, T6 et T8T9.

Les adénopathies étaient présentes chez deux patients (4.7\%), siégeant au niveau inguinal et axillaire dans un cas.

Une porte d'entrée était retrouvée, toutes dans l'arthrite septique, elle est cutanée dans 54\% de cas, génitale dans 33\% de cas et ORL dans 13\% de cas.

\section{Facteurs favorisants}

Le terrain prédisposant était : les traumatismes dans 9 cas (21.4\%), dont quatre (9.6\%) étaient en rapport avec un accident de la voie publique (AVP) ; Un geste chirurgical au niveau du site infectieux a été noté chez cinq patients (11.9\%), l'antécédent de chirurgie avec pose de matériel dans 4 cas (9.5\%), l'alcoolisme dans 4 cas (9.5\%), le diabète sucré, la corticotherapie , l'insuffisance rénale, la drepanocytose, le VIH /SIDA, le lupus erythemateux sytemique et la tuberculose pulmonaire dans 1 cas chacun. 


\section{Données paracliniques \\ Difficultés diagnostiques \\ Données biologiques}

La numération formule sanguine était réalisée chez 32 patients (76.1\%), elle montrait chez 27 patients (64.3\%) les anomalies suivantes: une hyperleucocytose à polynucléaires neutrophiles avec un nombre moyen de globules blancs de 12563 cellules par $\mathrm{mm}^{3}$ avec des extrêmes de 3400 et 34930 cellules par mm3 ; une anémie inflammatoire chez deux patients (4.7\%) avec un taux moyen d'hémoglobine de 9,5 g/dl avec des extrêmes de 3,5 et $15 \mathrm{~g} / \mathrm{dl}$.

La protéine C-réactive était réalisée chez 28 patients (66.7\%), elle était élevée chez 27 patients (64.3\%). Avec une CRP moyenne de $74 \mathrm{mg} / \mathrm{l}$ avec des extrêmes de 0 et $444 \mathrm{mg} / \mathrm{l}$. Une vitesse de sédimentation n'a été réalisée que chez 18 patients (42.3\%), la valeur moyenne était de 78 millimètres à la première heure avec des extrêmes de 0 et $150 \mathrm{~mm}$.

\section{Prelevements bacteriologiques}

Les prélèvements bacteriologiques ont été faits dans 25 cas (59.5\%) sur 42 patients avec un delai moyen de realisation de l'acte de 5,8 jours avec des extremes de 2 et 11 jours. Ils étaient faits au niveau de la porte d'entrée dans 11 cas (26.2\%) ; les urines (ECBU) dans 6 cas (14.3\%) ; le liquide articulaire dans 5 cas (11.9\%) ; les hémocultures dans 2 cas $(4.7 \%)$ et la biopsie osseuse dans 1 cas (2.4\%). La culture était positive dans 13 cas (30.9\%).

Le streptocoque était le germe le plus retrouvé ( 5 fois) suivi du staphylocoque (3 fois) (Figure 2).

\section{Imagerie}

Radiographie standard : elle a été prescrites chez tous les patients à l'admission. Les anomalies ont été notées chez 39 patients (92.8\%). L'étude analytique des signes radiologiques par affection musculosquelettique est illustrée dans le Tableau III.

\section{Imagerie en coupe}

L'imagerie en coupe était realisée dans $14.3 \%$ cas avec un delai moyen de realisation de 6,1 jours avec des extremes de 4 et 10 jours .

\section{Imagerie par résonance magnetique}

L'imagerie par résonance magnétique sans injection du produit de contraste a été réalisée chez quatre patients. Les anomalies retrouvées au cours d'une spondylodiscite inctieuse d'origine tuberculeuse étaient : un hyposignal T1 des disques et corps vertébraux C7-T2, hypersignal T2 des disques avec érosion des plateaux vertébraux en miroir C7-T1 et un hypersignal en STIR 
dans un cas ; un hyposignal T1, hypersignal T2 aux étages T11-T12 et T12L1 et un hypersignal en STIR dans un cas ; une compression médullaire dans 2 cas ; une luxation de la rotule et une sacroiliite gauche dans un cas chacun au cours d'arthrite septique.

\section{Scanner}

La tomodensitométrie a été réalisée chez deux patients, montrant : une lyse osseuse et une collection des parties molles au cours d'une coxite et une lyse osseuse, une collection des parties molles avec une compression médullaire dans la spondyloscite infectieuse d'origine tuberculeuse.

L'échographie a été réalisée chez six patients (14.3\%) ; il s'est agi d'une échographie articulaire dans 5 cas et des parties molles dans un cas. Les anomalies retrouvés étaient :une collection des parties molles dans 3 cas ,un épanchement intraarticulaire dans 2 cas et une hyperhémie de la membrane synoviale dans 1 cas.

\section{Données thérapeutiques Difficultés therapeutiques}

La prise en charge était multidisciplinaire associant des rhumatologues, des traumatologues, des infectiologues et les kinésithérapeutes.

L'antibiothérapie était prescrite chez tous les patients, associant une bi ou tri antibiothérapie probabiliste contre les germes banals chez 31 patients, adaptée après l'antibiogramme chez 13 patients ,une antibiothérapie spécifique pour les infections présumées à bacille de Koch (BK) chez 11 patients selon le protocole national de lutte contre la tuberculose EHRZ (E= ethambutol, $\mathrm{H}=$ isoniazide, $\mathrm{R}=$ rifampicine, $\mathrm{Z}=$ pyrazinamide) pendant 12 mois reparti en 2 périodes : 1ère période EHRZ pendant 2 mois et 2ème période RH à partir du 3ème mois jusqu'à au moins 12 mois.

La durée moyenne du traitement était de 60 jours avec les extrêmes de 21 et 365 jours.

La chirurgie a été réalisée chez 22 patients et l'immobilisation a été faite chez 20 patients.

\section{Difficultés évolutives}

La durée moyenne d'hospitalisation était de 19,07 jours avec des extrêmes de 3 et 92 jours. L'évolution était favorable pendant la période d'hospitalisation chez 38 patients (90.5\%), marquée par la régression des signes inflammatoires locaux, l'apyrexie, la régréssion du syndrome inflammatoire biologique et la stabilisation des signes radiologiques. Chez deux patients (4.7\%), l'évolution était défavorable, marquée par la survenue d'un décès par choc septique et une suppuration respectivement dans un 
cas.L'évolution à moyen terme (après la sortie) : 39 patients (92.8\%) sont suivis régulièrement, une récidive et une ankylose ont été notées respectivement dans un cas.Une arthrodèse a été réalisée chez un patient et 3 patients ont été perdus de vue (7.1\%).

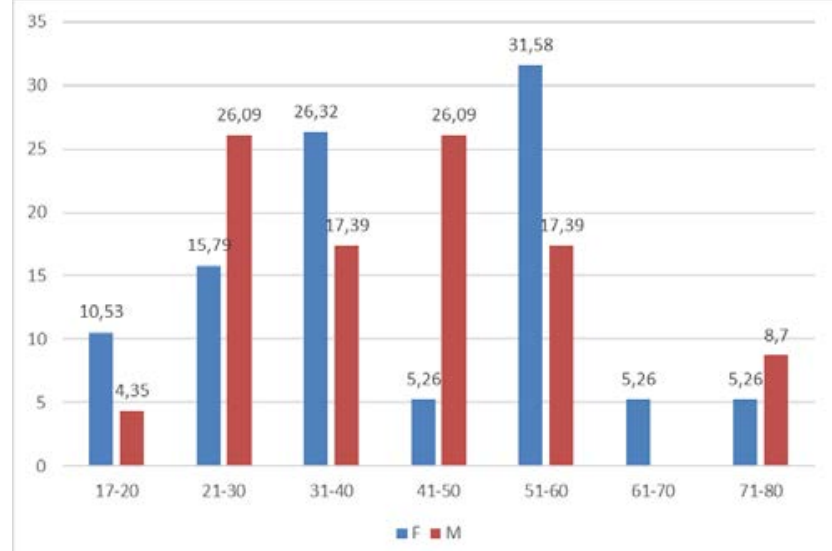

Figure 1. Répartition des patients en fonction de l’âge et du genre

Tableau I. Répartition des patients en fonction des pathologies

\begin{tabular}{|c|c|c|}
\hline Pathologies & Nombre de cas & $\mathbf{\%}$ \\
\hline Ostéomyélite/Ostéite & 13 & 30.9 \\
\hline Arthrite septique & 12 & 28.5 \\
\hline Spondylodiscite infectieuse & 9 & 21.4 \\
\hline Myosite & 4 & 9.6 \\
\hline Infection sur matériel & 4 & 9.6 \\
\hline Total & 42 & 100 \\
\hline
\end{tabular}

Tableau II. Signes locaux

\begin{tabular}{|c|c|c|}
\hline Localisation & Effectif (n) & $\mathbf{\%}$ \\
\hline Au Niveau des Segments des Membres & & \\
\hline Douleur & 17 & 100 \\
\hline Tuméfaction & 17 & 100 \\
\hline Plaie & 8 & 47.1 \\
\hline Fistule & 6 & 35.3 \\
\hline Au Niveau des Articulations Périphériques & & \\
\hline Douleur & 12 & 100 \\
\hline Raideur & 11 & 91.7 \\
\hline Tuméfaction & 7 & 58.3 \\
\hline Choc rotulien & 7 & 58.3 \\
\hline Luisance & 7 & 58.3 \\
\hline Rougeur & 7 & 58.3 \\
\hline Au Niveau Axial & 5 & 41.7 \\
\hline Douleur & & 100 \\
\hline Raideur & 9 & 100 \\
\hline Contracture des muscles paravertébraux & 9 & 91.7 \\
\hline
\end{tabular}




\begin{tabular}{|c|c|c|}
\hline Gibbosité & 4 & 44.4 \\
\hline Au Niveau Musculaire & & \\
\hline Tuméfaction & 4 & 100 \\
\hline Rougeur & 4 & 100 \\
\hline Luisance & 4 & 100 \\
\hline Augmentation de la chaleur locale & 4 & 100 \\
\hline Douleur & 4 & 100 \\
\hline
\end{tabular}

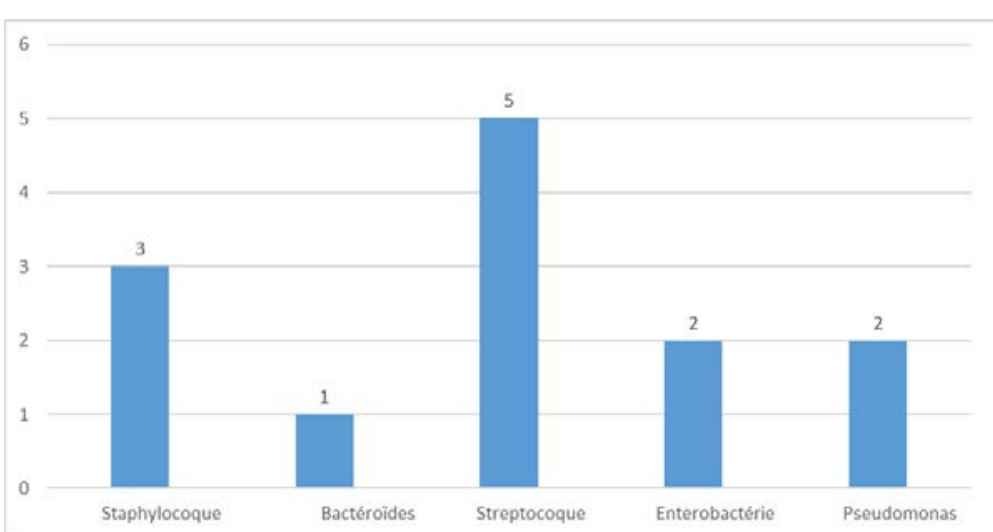

Figure 2. Répartition des espèces bactériennes isolées

Tableau III. Principaux signes radiologiques observés au cours des différentes pathologies

\begin{tabular}{|c|c|c|}
\hline & Effectif (n) & $\%$ \\
\hline \multicolumn{3}{|l|}{ Ostéomyélite/Ostéite } \\
\hline Apposition périostée & 7 & 17.9 \\
\hline Séquestre & 6 & 15.3 \\
\hline Lyse osseuse & 6 & 15.3 \\
\hline Ostéocondensation & 6 & 15.3 \\
\hline Epaississement de la corticale & 4 & 10.3 \\
\hline Epaississement des parties molles & 2 & 5.1 \\
\hline Abcès de Brodi & 1 & 2.5 \\
\hline \multicolumn{3}{|l|}{$\begin{array}{l}\text { Arthrite septique } \\
\end{array}$} \\
\hline Pincement de l'interligne articulaire & 9 & 23.1 \\
\hline Déminéralisation osseuse & 7 & 17.9 \\
\hline Géodes & 4 & 10.3 \\
\hline Epaississement des parties molles & 3 & 7.7 \\
\hline Erosions & 1 & 2.5 \\
\hline \multicolumn{3}{|l|}{$\begin{array}{l}\text { Spondylodiscite } \\
\end{array}$} \\
\hline Effacement du liséré cortical & 5 & 12.8 \\
\hline Déminéralisation osseuse & 5 & 12.8 \\
\hline Pincement discal & 4 & 10.3 \\
\hline Fuseau paravertebral & 1 & 2.5 \\
\hline Erosion & 1 & 2.5 \\
\hline Tassement & 1 & 2.5 \\
\hline Géode & 1 & 2.5 \\
\hline Infection sur matériel & & \\
\hline
\end{tabular}




\begin{tabular}{|c|c|c|}
\hline Séquestre & 2 & 5.1 \\
\hline Epaississement des parties molles & 1 & 2.5 \\
\hline Déminéralisation osseuse & 1 & 2.5 \\
\hline Réaction périostée & 1 & 2.5 \\
\hline Myosite & 4 & 100 \\
\hline Epaississement des parties molles & 4 & \\
\hline
\end{tabular}

\section{Discussion}

\section{Fréquence}

Du 1er Janvier 2017 au 30 Septembre 2020, nous avons recensé 42 cas d'infections musculosquelettiques dans les services de Rhumatologie et Traumatologie-Orthopédie du Centre Hospitalier Universitaire de Brazzaville, soit une fréquence hospitalière de $1.9 \%$. Le programme de médicalisation des systèmes d'information (PMSI) en France en 2008 rapportait une fréquence hospitalière de $0.22 \%$ des infections ostéoarticulaires (Grammatico et al., 2013). Notre fréquence était supérieure à celle du PMSI, cela pourrait s'expliquer par la taille de leur échantillon (16 millions d'hospitalisation et 36091 cas d'IOA).

\section{Sexe Et Age}

Notre étude avait rétrouvé une prédominance masculine ( $54.8 \%$ avec un sex-ratio $=1,2)$. Cette prédominance masculine est classiquement retrouvée dans la littérature (Habett EL khalil au Maroc en 2018) rapportait une prédominance masculine (79.41\%); il en est de même pour Grammatico et al en France en 2016 (63.8\%) et Sangare et al au Mali en 2008 (80.77\%) (Habett EL khalil, 2018; Grammatico et al., 2012; Sangare et al., 2008). Les infections musculosquelettiques touchent toutes les tranches d'âge (Asseray et al., 2012), dans notre étude l'âge moyen était de 41,6 ans avec des extrêmes de17 et 77 ans. La tranche d'âge la plus représentée était celle de 51-60 ans avec 23,8\% des cas. Nos résultats sont inferieurs à ceux de Guillon en France qui trouvait 56,5\% des cas entre 50-69 ans avec une prévalence augmentant avec l'âge (Grammatico et al., 2012).Cela pourrait s'expliquer par le vieillissement de la population vulnerable aux infections.

\section{Facteurs favorisants}

Plusieurs facteurs prédisposent certaines personnes à développer les infections musculosquelettiques notamment le diabète sucré, l’âge avancé (supérieur ou égal à 60 ans), la polyarthrite rhumatoïde, les connectivites, la corticothérapie, l'insuffisance rénale, l'immunodépression, l'accident de la voie publique (AVP), les hémoglobinopathies, la toxicomanie et les gestes invasifs (chirurgie-infiltration) sur une articulation etc (Asseray et al., 2012). Dans notre série, les deux principaux facteurs de risque favorisant les infections musculosquelettiques étaient le traumatisme (21.4\% des cas) et la 
drépanocytose (21.4\% des cas). Habett El khalil au Maroc a démontré une forte implication des traumatismes dans la survenue des infections ostéoarticulaires (Habett El khalil, 2018).

\section{Difficultés Diagnostiques}

Le diagnostic des infections musculosquelettiques se fait sur la base des données cliniques, biologiques et d'imagerie (Weston et al., 1999). Dans notre étude, le diagnostic s'est plus fait sur la base des données cliniques et radiologiques. Les examens bacteriologiques n’ont été réalisés que chez 25 patients, la culture n'a isolé que 13 fois le germes. Cela pourrait s'expliquer par la faible accessibilité au plateau technique, la précarité de nos patients ne pouvant pas faire face au examens avec un cout élévé et l'automédication qui est une situation fréquente dans notre contexte pouvant décapiter le germe, allongeant ainsi le délai de consultation. Il est décrit dans la littérature que Staphylocuque doré est le germe fréquemment retrouvé (Monka et al., 2018; Grammatico et al., 2012; Patel et al., 1997). Dans notre étude le streptocoque était le germe le plus retrouvé ( 5 fois) suivi du staphylocoque (3 fois). Cette différence peut être expliquée par le fait que l'analyse bactériologique n'a pas été faite chez tous nos patients. Le membre pelvien était le membre le plus atteint 29 fois. Monka au Congo en 2019 rapportait une atteinte du membre pelvien 17 fois sur 22 cas. Nos résultats sont supérieurs, cette différence pourrait s'expliquer par le fait que l'étude de Monka n'a porté que sur les ostéomyélites (Monka et al., 2018).

\section{Difficultés thérapeutiques}

Le traitement était instauré sur la base des arguments présomptifs alors qu'il devrait se faire sur la base des résultats bactériologiques comme décrit dans la littérature (Dubost et al., 2000; Gupta et al., 2001; Ledantec et al., 1999; Studahl et al., 1994). Dans notre étude, l'antibiothérapie dans la majorité des cas était probabiliste et le demeurait jusqu'à la guérison. Ceci pourrait s'expliquer par l'insuffisance des moyens financiers à réaliser les examens paracliniques d'orientation.

\section{Difficultés évolutives}

Nos patients étaient admis à un stade chronique (64.3\%), le délai moyen de consultation était de 27 jours avec des extrêmes de 5 et 223 jours, ce qui rendait la prise en charge laborieuse.

La durée moyenne d’hospitalisation dans notre serie était de 19,07 jours. Cette durée d'hospitalisation est légèrement superieure à celle rapportée par le PMSI (17,4 jours) (Grammatico et al., 2012). Cela pourrait s'expliquer par la durée d'attente des examens préscrits et la précarité de nos patients ne pouvant faire face rapidement à l'achat des médicaments. L'évolution était 
émaillée de récidives, des complications. Cela pourrait s'expliquer par la précarité de nos patients et leur croyance, nombreux avaient recours initialement aux traitements traditionnels comme raportait Sangare A et al au Mali en 2008 (Sangare et al., 2008).

\section{Conclusion}

De cette étude hospitalière portant sur une période de 3 ans et 8 mois, il ressort les faits suivants :

Les infections musculosquelettiqu sont fréquentes dans les pays en voie de déveleppement. La prise en charge adéquate des infections musculosquelettiques se heurte à des difficultés inhérentes aux conditions d'exercice en milieu hospitalier sous équipé. En effet, le retard diagnostic, l'insuffisance du plateau technique ne permettent pas de réunir les arguments paracliniques contributifs à la certitude du diagnostic et à une antibiothérapie précoce et adaptée. De même, le niveau socioéconomique de nos patients ne leur permet pas de faire face aux examens paracliniques à cause de leur coût élevé et les difficultés de collecte des données à cause de l'inaccessibilité infrastructurelle dûes à la prise en charge des patients COVID positif. Malgré tout, la guérison a été obtenue le plus souvent par une prise en charge médicochirurgicale avec une stratégie antibiotique dominée par une biantibiothérapie probabiliste au prix de séquelles fonctionnelles dépendant de l'importance de dégradation articulaire et osseuse pré-thérapeutique, avec un suivi qui reste difficile dans notre contexte.

\section{References}

1. Asseray, N., Dary, M., \& Potel, G. (2012). Arthrites septiques aiguës de l'adulte Mécanismes physiopathologiques des arthrites septiques, stratégie diagnostique et thérapeutique à mettre en oeuvre. Urgences infectieuses; $53: 1-8$.

1. Bussiere, F. \& Beaufils, P. (1999). Arthroscopic management of pyoarthritis of the adult knee. Report of 16 cases. Rev Chir Orthop; 85: 803-10.

2. Biléckot, RR., Miakoundoba, RC., \& Yala, F. (2006). Microbiology and prognosis of septic arthritisin Brazzaville. Joint Bone Spine 73 ; 570-8.

3. Dubost, JJ., Soubrier, M., \& Sauvezie, B. (2000). Pyogenic arthritis in adults. Joint Bone Spine; 67: 11-21.

4. Faraj, AA., Omonbude, OD., \& Godwin, B. (2002). Gram staining in the diagnosis of acute septic arthritis. Acta Orthop Belg; 68: 388-91.

5. Gupta, MN., Sturrock, RD., \& Field, M. (2001). A prospective 2 year study of 75 patients with adult-onset septic arthritis. Rheumatology; 40: 24-30. 
6. Grammatico, GL., Baron, S., Gettner, S., Lecuyer, AI., Gaborit, C., Rosset, P. et al. (2013). Surveillance hospitalière des infections ostéoarticulaires en France: analyse des données médicoadministratives, PMSI 2008. Bulletin Epidémiologique Hebdomadaire; 4-5 : 39-44.

7. Grammatico, GL., Baron, S., Gettner, S., Lecuyer, AI., Gabout, C., Rosset, P. et al. (2012). Bone and joint infection in hospitalized patients in France, 2008: clinical and economic outcomes. J Hosp Inf Sept ; $82:$ 40-8.

8. Habett El khalil (2018). Profil épidémiologique des infections ostéoarticulaires. Marrakech: Faculté de médecine et de pharmacie. Thèse de doctorat en Médecine, Maroc; $\mathrm{n}^{\circ} 155$ : 122p.

9. Houzou, P., Kakpovi, K., Fianyo, E., Viwalé, E S.,Tagbor, K C., Landoh, D E. et al. (2017). Profil des IOA en consultation Rhumatologique au CHU-Kara (Togo). European Scientific Journal september; 251-7.

10. Jerosh, J., Hoffstetter, I., Schroder, M., \& Castro, WH. (1995). S antibioticeptic arthritis: arthroscopic management with local antibiotic treatment. Acta Orthop Belg; 61: 126-34.

11. Jerosh, J. \& Prymka, M. (1998). Arthroscopic therapy of septic arthritis surgical technique and results. Unfall Chirurg; 101: 454-60.

12. Ledantec, L., Maury, F., \& Flipo, RM. (1996). Peripheral pyogenic arthritis. A study of one hundred seventy-nine cases. Rev Rhum Engl Ed; 63: 103-10.

13. Lamini, NNE., Moyikoua, R., Moussounda, MGS., Ntsiba, H., \& Biléckot, R. (2019). Aspects morphologiques du mal de Pott chez l'adulte congolais à Brazzaville. Health Sci Dis September - October ; 20 (5) : 64-7.

14. Malghem, J., Mosseray, A., VandeBerg, B., Lebon, C., \& Maldague, B. (1997). Radiologic aspects of the loosening of cemented hip prostheses : mechanical, septic or granulomatous etiology ? J Belge Radiol ; 80(4): 173-84.

15. Monka, M., Mboutol, MC., Massamba, MD., Note, MM., Ngatsé, OA., \& Moyikoua, A. (2018). Ostéomyélites chroniques de l'adulte à Brazzaville. Health Sci Dis April - May - June ;19 (2) : 127-30.

16. Ntsiba, H., Bazébissa, R., Lamini, N., \& Yala, F. (2003). Cent cas d'arthrites septiques du genou en zone tropicale. Bull Soc Pathol Exot; 244-6.

17. Moyikoua, A., Kaya, JM., Ondzotto, JM., \& Pena-Pitra, B. (1993). Complications septiques des ostéosynthèses des membres. A propos de 402 interventions. Méd Afr Noire; 40 (12) :723-6. 
18. Patel, SR., Olenginski, TP., Perruquet, J., \& Harrington, TM. (1997). Clinical features and predisposing conditions. J Rheumatol; 24: 17348.

19. Studahl, M., Bergman, B., Kalebo, P., \& Lindberg, J. (1994). Septic arthritis of the knee: a 10 year review and long term follow up using a new scoring system. Scand J infect Dis ; 26: 85-93.

20. Sangare, A., Alwata, I., Sidibe, S., Macalou, M., \& Toure, AA. (2008). Les ostéites dans les services d'Orthopédie et de traumatologie de l'hopital Gabriel Touré de Bamako. Mali medical ; 23(1) : 27-30.

21. Visposeara, JL., Barthel, T., Schmitz, H., \& Eulert, J. (2002). Arthroscopic treatment of septic joints : prognostic factors. Arch orthop Trauma Surg; 122: 204-11.

22. Weston, V., Jones, A., Bradbury, N., Fawthrop, F., \& Doherty, M. (1999). Clinical features and out-come of septic arthritis in a single UK Health District 1982-1991. Ann Rheum Dis ;58: 214-9. 